Pacific

Journal of

Mathematics

COMPLETE CONTRACTIVITY OF MAPS ASSOCIATED WITH THE ALUTHGE AND DUGGAL TRANSFORMS

Ciprian Foiaş, Il Bong Jung, Eungil Ko, and Carl Pearcy 


\title{
COMPLETE CONTRACTIVITY OF MAPS ASSOCIATED WITH THE ALUTHGE AND DUGGAL TRANSFORMS
}

\author{
Ciprian Foiaş, Il Bong Jung, Eungil Ko, and Carl Pearcy
}

For an arbitrary operator $T$ on Hilbert space, we study the maps $\widetilde{\Phi}: f(T) \rightarrow f(\widetilde{T})$ and $\widehat{\Phi}: f(T) \rightarrow f(\widehat{T})$, where $\widetilde{T}$ and $\widehat{T}$ are the Aluthge and Duggal transforms of $T$, respectively, and $f$ belongs to the algebra $\operatorname{Hol}(\sigma(T))$. We show that both maps are (contractive and) completely contractive algebra homomorphisms. As applications we obtain that every spectral set for $T$ is also a spectral set for $\widehat{T}$ and $\widetilde{T}$, and also the inclusion $W(f(\widetilde{T}))^{-} \cup W(f(\widehat{T}))^{-} \subset W(f(T))^{-}$relating the numerical ranges of $f(T), f(\widetilde{T})$, and $f(\widehat{T})$.

\section{Introduction.}

Let $\mathcal{H}$ be an arbitrary separable, complex Hilbert space whose dimension satisfies $2 \leq \operatorname{dim} \mathcal{H} \leq \aleph_{0}$, and denote by $\mathcal{L}(\mathcal{H})$ the algebra of all bounded linear operators on $\mathcal{H}$. If $T \in \mathcal{L}(\mathcal{H})$ we shall always write, without further mention, $T=U P$ to be the unique polar decomposition of $T$ ( so $P=|T|=$ $\left(T^{*} T\right)^{\frac{1}{2}}$ and $U$ is the appropriate partial isometry satisfying $\operatorname{ker} U=\operatorname{ker} T$ and $\left.\operatorname{ker} U^{*}=\operatorname{ker} T^{*}\right)$. Also we write, as usual, $\sigma(T)$ for the spectrum of such a $T$.

In this paper we consider the following two transforms of an arbitrary $T=U P$ in $\mathcal{L}(\mathcal{H}):$

(a) the Aluthge transform $\widetilde{T}:=P^{\frac{1}{2}} U P^{\frac{1}{2}}$, which was first studied in [1] and which has been studied extensively since, mostly in the context of $p$ hyponormal operators. In particular, some of the present authors studied the map $T \rightarrow \widetilde{T}$ for an arbitrary $T$ in $\mathcal{L}(\mathcal{H})$ in [4], [5] and [6].

We obtained in [4] various spectral identities and showed that if $T$ is a quasiaffinity, then the invariant subspace lattice $\operatorname{Lat}(T)$ is nontrivial if and only if $\operatorname{Lat}(\widetilde{T})$ is nontrivial, and the same is true of the hyperinvariant subspace lattices HLat $(T)$ and HLat $(\widetilde{T})$. Furthermore, we showed that the map $T \rightarrow \widetilde{T}$ is $(\|\|,\|\|)$ continuous at every $T$ in $\mathcal{L}(\mathcal{H})$ with closed range, and we conjectured that for an arbitrary $T$ in $\mathcal{L}(\mathcal{H})$, where $\mathcal{H}$ is finite dimensional, the sequence $\left\{\widetilde{T}^{(n)}\right\}$ of Aluthge iterates of $T$, defined by $\widetilde{T}^{(0)}=T$ and $\widetilde{T}^{(n+1)}=\left(\widetilde{T}^{(n)}\right)^{\sim}$ for $n \in \mathbb{N}$, converges to a normal operator. Our study was continued in [5], in which we showed that if $T$ is an arbitrary operator 
in $\mathcal{L}(\mathcal{H})$ such that the spectral picture $\mathrm{SP}(T)$ of $T$ (or that of $\widetilde{T}$; cf. [9]) contains no pseudoholes, then $\operatorname{SP}(T)=\operatorname{SP}(\widetilde{T})$, and we derived connections between $T$ and $\widetilde{T}$ as consequences of this equality (e.g., $T$ is quasitriangular if and only if $\widetilde{T}$ is quasitriangular).

Moreover, in [6] we pursued the study of the sequence $\left\{\widetilde{T}^{(n)}\right\}$ of Aluthge iterates of an arbitrary $T$ in $\mathcal{L}(\mathcal{H})$, and we established the validity of $[\mathbf{4}$, Conjecture 1.11] in certain special cases. We also initiated a study of the backward Aluthge iterates of an arbitrary $T$ in $\mathcal{L}(\mathcal{H})$.

(b) The Duggal transform $\widehat{T}:=P U$ (named after Professor B. P. Duggal, who suggested its study to us), has been studied very little.

We will explore below various relations between $T, \widehat{T}$, and $\widetilde{T}$ by studying maps between the Riesz-Dunford algebras associated with these operators. It is well-known (and not difficult to see, cf. [4]) that

$$
\sigma(T)=\sigma(\widehat{T})=\sigma(\widetilde{T}), \quad T \in \mathcal{L}(\mathcal{H}) .
$$

In what follows, when some $T$ in $\mathcal{L}(\mathcal{H})$ is under consideration, we denote by $\operatorname{Hol}(\sigma(T))$ the algebra of all complex-valued functions which are analytic on some neighborhood of $\sigma(T)$, where linear combinations and products in $\operatorname{Hol}(\sigma(T))$ are defined (with varying domains) in the obvious way. Moreover, the (Riesz-Dunford) algebra $\mathcal{A}_{T} \subset \mathcal{L}(\mathcal{H})$ is defined as

$$
\mathcal{A}_{T}=\{f(T): f \in \operatorname{Hol}(\sigma(T))\},
$$

(where $f(T)$ is defined by the Riesz-Dunford functional calculus). As our main theorem (Th.1.1) shows, it is possible to obtain useful information about $\widetilde{T}$ and $\widehat{T}$ by studying maps between the algebras $\mathcal{A}_{T}, \mathcal{A}_{\widetilde{T}}$, and $\mathcal{A}_{\widehat{T}}$.

Theorem 1.1. For every $T$ in $\mathcal{L}(\mathcal{H})$, with $\widehat{T}, \widetilde{T}$, and $\operatorname{Hol}(\sigma(T))$ as defined above:

a) The maps $\widehat{\Phi}: \mathcal{A}_{T} \rightarrow \mathcal{A}_{\widehat{T}}$ and $\widetilde{\Phi}: \mathcal{A}_{T} \rightarrow \mathcal{A}_{\widetilde{T}}$ defined by

$$
\widehat{\Phi}(f(T))=f(\widehat{T}), \quad \widetilde{\Phi}(f(T))=f(\widetilde{T}), \quad f \in \operatorname{Hol}(\sigma(T)),
$$

are well-defined contractive algebra homomorphisms; in particular,

$$
\max \{\|f(\widehat{T})\|,\|f(\widetilde{T})\|\} \leq\|f(T)\|, \quad f \in \operatorname{Hol}(\sigma(T)) .
$$

b) More generally, the maps $\widehat{\Phi}$ and $\widetilde{\Phi}$ in a) are completely contractive, meaning that for every $n \in \mathbb{N}$ and every $n \times n$ matrix $\left(f_{i j}\right)$ with entries from $\operatorname{Hol}(\sigma(T))$,

$$
\max \left\{\left\|\left(f_{i j}(\widehat{T})\right)\right\|,\left\|\left(f_{i j}(\widetilde{T})\right)\right\|\right\} \leq\left\|\left(f_{i j}(T)\right)\right\| .
$$

c) Every spectral set [ $M$-spectral set (for fixed $M>1$ )] for $T$ is also a spectral set [respectively, $M$-spectral set] for both $\widehat{T}$ and $\widetilde{T}$. 
d) If $W(S)$ denotes the numerical range of an operator $S$ in $\mathcal{L}(\mathcal{H})$, then $W(f(\widehat{T}))^{-} \cup W(f(\widetilde{T}))^{-} \subset W(f(T))^{-}, \quad f \in \operatorname{Hol}(\sigma(T))$,

and, moreover, if $T$ belongs to some class $\mathcal{C}_{\rho}$, then $\widehat{T}$ and $\widetilde{T}$ belong to $\mathcal{C}_{\rho}$ also (see $[\mathbf{7}, \mathrm{p} .45]$ for the definition of these classes).

The result d) verifies (except for the closure bar) an earlier conjecture of the authors [4, Conjecture 1.9] and extends recent work of T. Yamazaki [10], who showed that $W(\widetilde{T}) \subset W(T)$ if $T$ acts on a finite dimensional space and that $w(\widetilde{T}) \subset w(T)$ in complete generality, where, of course, $w(T)$ denotes the numerical radius of $T$.

The proof of a) of Theorem 1.1 requires some lemmas and will be given in Section 2. On the other hand, c) follows immediately from a) and the definitions of spectral and $M$-spectral sets, so no proof of c) need be given. The result d) is also an easy consequence of c), but a proof will be given in Section 3. Finally, b) will be established in Sections 4 and 5.

\section{Proof of Theorem $1.1 \mathrm{a})$.}

It is obvious that the maps $\widehat{\Phi}$ and $\widetilde{\Phi}$ are algebra homomorphisms provided that they are well-defined, and this will follow from the inequalities (1). Thus it sufficies to establish (1). As noted above, the proof depends upon several lemmas. The first of these summarizes some easy calculations, so no proof need be given.

Lemma 2.1. For every $T=U P$ in $\mathcal{L}(\mathcal{H})$, we have
a) $P T=\widehat{T} P$,
b) $T U=U \widehat{T}$
c) $P^{\frac{1}{2}} T=\widetilde{T} P^{\frac{1}{2}}$, and
d) $P^{\frac{1}{2}} \widetilde{T}=\widehat{T} P^{\frac{1}{2}}$.

Lemma 2.2. For every $T=U P$ in $\mathcal{L}(\mathcal{H})$ and every $f \in \operatorname{Hol}(\sigma(T))$, we have
a) $P f(T)=f(\widehat{T}) P$,
b) $f(T) U=U f(\widehat{T})$,
c) $P^{\frac{1}{2}} f(T)=f(\widetilde{T}) P^{\frac{1}{2}}$, and
d) $P^{\frac{1}{2}} f(\widetilde{T})=f(\widehat{T}) P^{\frac{1}{2}}$.

Proof. If $f$ is a polynomial, the desired relations follow from Lemma 2.1 by trivial calculations. Next suppose that $f=p / q$ is a rational function, where $p$ and $q$ are polynomials such that $q$ doesn't vanish on $\sigma(T)$. Then $q(T)$ and $q(\widehat{T})$ are invertible (for example) and the equation $P q(T)=q(\widehat{T}) P$ yields immediately $P q(T)^{-1}=q(\widehat{T})^{-1} P$ (for example), so the desired relations are valid for all rational functions in $\operatorname{Hol}(\sigma(T))$. The lemma now results 
easily from Runge's theorem and the well-known continuity properties of the Riesz-Dunford functional calculus (cf., e.g., [2, Prop. 17.26]).

Lemma 2.3. For every $T=U P$ in $\mathcal{L}(\mathcal{H})$ and every $f \in \operatorname{Hol}(\sigma(T)), f(\widehat{T})$ is the (orthogonal) direct sum

$$
f(\widehat{T})=\left.E U^{*} f(T) U E\right|_{(\operatorname{ker} T)^{\perp}} \oplus f(0) 1_{\mathrm{ker} T},
$$

where $E$ is the (orthogonal) projection $U^{*} U$ on $(\operatorname{ker} T)^{\perp}$, and, consequently,

$$
\|f(\widehat{T})\| \leq\|f(T)\| .
$$

Proof. If $T$ has trivial kernel, then $U$ is an isometry, and thus $E=1_{\mathcal{H}}$ and $f(\widehat{T})=U^{*} f(T) U$, so (2) and (3) are satisfied. Thus we may suppose that $0 \in \sigma(T)$, and hence $f$ is analytic at $z=0$ and $f(0) \in \sigma(f(T))$. An easy calculation shows that $E \widehat{T}=\widehat{T} E=\widehat{T}$, and thus (by writing $f(z)=$ $f(0)+z g(z)$, where $g \in \operatorname{Hol}(\sigma(T)))$ that $f(\widehat{T}) E=E f(\widehat{T})$. Hence

$$
\begin{aligned}
f(\widehat{T}) & =\left.E f(\widehat{T}) E\right|_{(\operatorname{ker} T)^{\perp}} \oplus f(0) 1_{\mathrm{ker} T} \\
& =\left.E U^{*} f(T) U E\right|_{(\operatorname{ker} T)^{\perp}} \oplus f(0) 1_{\mathrm{ker} T},
\end{aligned}
$$

from b) of Lemma 2.2, and thus

$$
\|f(\widehat{T})\| \leq \max \{\|f(T)\|,|f(0)|\}=\|f(T)\| .
$$

Lemma 2.4. For every $T=U P$ in $\mathcal{L}(\mathcal{H})$ such that $P$ has trivial kernel (which implies, of course, that $U$ is an isometry) and for every $f \in$ $\operatorname{Hol}(\sigma(T)),\|f(\widetilde{T})\| \leq\|f(T)\|$.

Proof. Suppose first that $P$ is invertible. We use the fact from [3] that if $X \in \mathcal{L}(\mathcal{H})$ and $A$ and $B$ are positive semidefinite operators in $\mathcal{L}(\mathcal{H})$, then

$$
\left\|A^{\frac{1}{2}} X B^{\frac{1}{2}}\right\| \leq\|A X B\|^{\frac{1}{2}}\|X\|^{\frac{1}{2}} .
$$

We know from c) of Lemma 2.2 that

$$
f(\widetilde{T})=P^{\frac{1}{2}} f(T) P^{-\frac{1}{2}},
$$

so applying (4) with $A=P$ and $B=P^{-1}$ we obtain

$$
\|f(\widetilde{T})\|=\left\|P^{\frac{1}{2}} f(T) P^{-\frac{1}{2}}\right\| \leq\left\|P f(T) P^{-1}\right\|^{\frac{1}{2}}\|f(T)\|^{\frac{1}{2}} .
$$

Moreover, we know from a) of Lemma 2.2 that

$$
P f(T) P^{-1}=f(\widehat{T}),
$$

and thus (5) becomes

$$
\|f(\widetilde{T})\| \leq\|f(\widehat{T})\|^{\frac{1}{2}}\|f(T)\|^{\frac{1}{2}} \leq\|f(T)\|,
$$

by Lemma 2.3 , and the case in which $P$ is invertible is done. 
Now let $P$ be an arbitrary quasiaffinity. Define the sequence $\left\{Q_{n}\right\}$ of positive invertible operators by $Q_{n}=P+(1 / n) 1_{\mathcal{H}}$, and set $A_{n}=U Q_{n}$ (polar decomposition of $A_{n}$ ). Then $\widetilde{A}_{n}=\left(Q_{n}\right)^{1 / 2} U\left(Q_{n}\right)^{1 / 2}$, and since $\| Q_{n}-$ $P \| \rightarrow 0$ and $\left\|\left(Q_{n}\right)^{1 / 2}-P^{1 / 2}\right\| \rightarrow 0$, we obtain that $\left\|A_{n}-T\right\| \rightarrow 0$ and $\left\|\widetilde{A}_{n}-\widetilde{T}\right\| \rightarrow 0$. By what was proved above, we have $\left\|f\left(\widetilde{A}_{n}\right)\right\| \leq\left\|f\left(A_{n}\right)\right\|$ for all $n$ sufficiently large that $f\left(A_{n}\right)$ is defined. The result follows from the facts that $\left\|f\left(A_{n}\right)-f(T)\right\| \rightarrow 0$ and $\left\|f\left(\widetilde{A}_{n}\right)-f(\widetilde{T})\right\| \rightarrow 0$ (cf., for example, [2, Prop. 17.26]).

Lemma 2.5. For every $T=U P$ in $\mathcal{L}(\mathcal{H})$ such that

$$
\operatorname{dim}\left(\operatorname{ker} U^{*}\right) \geq \operatorname{dim}(\operatorname{ker} U)>0
$$

and for every $f \in \operatorname{Hol}(\sigma(T)),\|f(\widetilde{T})\| \leq\|f(T)\|$.

Proof. Choose a partial isometry $V$ such that the initial space of $V$ is $\operatorname{ker} U$ and the range of $V$ is a subspace of ker $U^{*}$. Define $A_{n}=T+(1 / n) V$ for $n \in \mathbb{N}$, and note that each $\left|A_{n}\right|$ is a quasiaffinity and that $\left\|A_{n}-T\right\| \rightarrow 0$. Since the polar decomposition of $A_{n}$ is $(U+V)\left|A_{n}\right|$ where $\left|A_{n}\right|$ is the direct sum $\left.P\right|_{(\operatorname{ker} T)^{\perp}} \oplus(1 / n) 1_{\mathrm{ker} T}$, it follows easily that $\left\|\widetilde{A}_{n}-\widetilde{T}\right\| \rightarrow 0$. From Lemma 2.4 we know that $\left\|f\left(\widetilde{A}_{n}\right)\right\| \leq\left\|f\left(A_{n}\right)\right\|$ and the result now follows as before from, e.g., [2, Prop. 17.26].

To complete the proof of a) of Theorem 1.1, it suffices, in view of Lemma 2.5, to deal with the case in which $T=U P$ and $U$ satisfies $\operatorname{dim}\left(\operatorname{ker} U^{*}\right)<$ $\operatorname{dim}(\operatorname{ker} U)$. Moreover, if $\operatorname{ker} U^{*}$ is nontrivial, by choosing a partial isometry $W$ whose range is $\operatorname{ker} U^{*}$ and whose initial space is a subspace of $\operatorname{ker} U$, and considering the sequence $\{T+(1 / n) W\}$ as in Lemma 2.5, we can reduce what is to be shown to the case in which $U$ is a nonunitary coisometry.

Lemma 2.6. For every $T=U P$ in $\mathcal{L}(\mathcal{H})$ such that $U$ is a nonunitary coisometry and for every $f \in \operatorname{Hol}(\sigma(T))$,

$$
\|f(\widetilde{T})\| \leq\|f(\widehat{T})\| \leq\|f(T)\| .
$$

Proof. Let $\mathcal{U}^{*}:=\{\bar{z}: z \in \mathcal{U}\}$, and let $\widetilde{f}$ be the analytic function on $\mathcal{U}^{*}$ defined, as usual, by $\widetilde{f}(z):=\overline{f(\bar{z})}, z \in \mathcal{U}^{*}$. Recall that in this situation, $\sigma\left(T^{*}\right) \subset \mathcal{U}^{*}$ and $f(T)^{*}=\widetilde{f}\left(T^{*}\right)$, so $\left\|\widetilde{f}\left(T^{*}\right)\right\|=\|f(T)\|$. Note that $\widehat{T}=P U$, and thus that $(\widehat{T})^{*}=U^{*} P$ with $U^{*}$ an isometry. Define, for $n \in \mathbb{N}$,

$$
S_{n}=U^{*}\left(P+(1 / n) 1_{\mathcal{H}}\right) .
$$

Since $P+(1 / n) 1_{\mathcal{H}}$ is invertible, (6) gives the polar decomposition of $S_{n}$, and hence

$$
\widetilde{S}_{n}=\left(P+(1 / n) 1_{\mathcal{H}}\right)^{\frac{1}{2}} U^{*}\left(P+(1 / n) 1_{\mathcal{H}}\right)^{\frac{1}{2}}, \quad n \in \mathbb{N} .
$$


It follows easily that $\left\|S_{n}-(\widehat{T})^{*}\right\| \rightarrow 0$ and that $\left\|\widetilde{S}_{n}-(\widetilde{T})^{*}\right\| \rightarrow 0$. Thus we have

$$
\|f(\widetilde{T})\|=\left\|\widetilde{f}\left((\widetilde{T})^{*}\right)\right\|=\lim _{n}\left\|\widetilde{f}\left(\widetilde{S}_{n}\right)\right\|,
$$

and

$$
\|f(\widehat{T})\|=\left\|\widetilde{f}\left((\widehat{T})^{*}\right)\right\|=\lim _{n}\left\|\widetilde{f}\left(S_{n}\right)\right\|
$$

(again by, e.g., [2, Prop. 17.26]). But Lemma 2.4 applies to each $S_{n}$, and thus

$$
\left\|\widetilde{f}\left(\widetilde{S}_{n}\right)\right\| \leq\left\|\widetilde{f}\left(S_{n}\right)\right\|, \quad n \in \mathbb{N} .
$$

Thus $\|f(\widetilde{T})\| \leq\|f(\widehat{T})\|$ and the other inequality follows from Lemma 2.3. This completes the proof of Theorem $1.1 \mathrm{a})$.

\section{Proof of Theorem $1.1 \mathrm{~d})$.}

In view of Theorem $1.1 \mathrm{c}$ ), which follows immediately from Theorem $1.1 \mathrm{a}$ ) as noted above, the first statement in d) follows trivially from the following known fact, and the other statements are immediate from Remarks 1, 2 and 3 on pp. 48 and 49 of [7]:

Proposition 3.1. For every $T \in \mathcal{L}(\mathcal{H}), W(T)^{-}$is the intersection of all closed half-planes $H$ containing $W(T)$ such that $H$ is a spectral set for $T$.

Proof. Since $W(T)^{-}$is convex, and is thus the intersection of all closed halfplanes containing $W(T)$, it suffices to show that if $H$ is any closed halfplane containing $W(T)$, then $H$ is a spectral set for $T$. By a harmless rotation and translation, we may suppose that $H$ is the closed right-halfplane $\{z: \operatorname{Re} z \geq 0\}$. Thus, writing $T=K+i L$, with $K$ and $L$ Hermitian, we see that $K$ is positive semidefinite, and therefore that the Cayley transform of $T$,

$$
c(T)=\left(T+1_{\mathcal{H}}\right)^{-1}\left(T-1_{\mathcal{H}}\right),
$$

is a contraction (cf., e.g., [7, p. 167]). Hence, by von Neuman's inequality, the closed unit disc $\mathbb{D}$ in $\mathbb{C}$ is a spectral set for $c(T)$, and thus, by taking inverse Cayley transforms, we obtain that $H$ is a spectral set for $T$, as desired.

\section{Complete contractivity of $\widehat{\Phi}$.}

In this section we prove the following theorem, which establishes a part of Theorem $1.1 \mathrm{~b})$ :

Theorem 4.1. For every $T$ in $\mathcal{L}(\mathcal{H})$, the map $\widehat{\Phi}: \mathcal{A}_{T} \rightarrow \mathcal{A}_{\widehat{T}}$ defined in Section 1 is completely contractive. 
Recall that this means, by definition, that for every $n \in \mathbb{N}$ and for every $n \times n$ matrix $\left(f_{i j}\right)$, where each $f_{i j} \in \operatorname{Hol}(\sigma(T))$, the inequality

$$
\left\|\left(f_{i j}(\widehat{T})\right)\right\| \leq\left\|\left(f_{i j}(T)\right)\right\|
$$

is satisfied. (Here of course, the $n \times n$ operator matrices in (8) act on the Hilbert space $\mathcal{H}^{(n)}$, the direct sum of $n$ copies of $\mathcal{H}$, and the norm indicated is the operator norm on $\mathcal{L}\left(\mathcal{H}^{(n)}\right)$.)

Proof of Theorem 4.1. Let $T \in \mathcal{L}(\mathcal{H})$, let $n \in \mathbb{N}$, and let $\left(f_{i j}\right)$ be an arbitrary $n \times n$ matrix with entries from $\operatorname{Hol}(\sigma(T))$. Then, with the notation as in Lemma 2.3, it is immediate from (2) that we have the matricial identity

$$
\left(f_{i j}(\widehat{T})\right)=\left(\left.E U^{*} f_{i j}(T) U E\right|_{(\operatorname{ker} T)^{\perp}}\right) \oplus\left(f_{i j}(0) 1_{\operatorname{ker} T}\right),
$$

where, of course, the first [second] matrix on the right acts on the space $\left\{(\operatorname{ker} T)^{\perp}\right\}^{(n)}\left[\right.$ respectively, $\left.\{\operatorname{ker} T\}^{(n)}\right]$. As in the proof of Lemma 2.3, if $T$ has trivial kernel, then $E=1_{\mathcal{H}}$ and $U$ is an isometry. Since it is obvious that the inequality

$$
\left\|\left(U^{*} f_{i j}(T) U\right)\right\| \leq\left\|\left(f_{i j}(T)\right)\right\|
$$

holds (the matrix on the left is the product of two diagonal matrices of norm at most one and the matrix on the right), it suffices to treat the case in which $\operatorname{ker} T \neq(0)$. Moreover, from (9), one sees easily that it is enough to show that

$$
\left\|\left(f_{i j}(0) 1_{\text {ker } T}\right)\right\| \leq\left\|\left(f_{i j}(T)\right)\right\| .
$$

Since $f_{i j}(0) \in \sigma\left(f_{i j}(T)\right), f_{i j}$ is analytic at $z=0$ for $i, j=1, \ldots, n$. Upon writing

$$
f_{i j}(z)-f_{i j}(0)=g_{i j}(z) z, \quad z \in \text { domain } f_{i j},
$$

we see that $g_{i j}(z) \in \operatorname{Hol}(\sigma(T))$ for $i, j=1, \ldots, n$, and hence we get the matricial identity

$$
\left(f_{i j}(T)-f_{i j}(0) 1_{\mathcal{H}}\right)=\left(g_{i j}(T) T\right)=\left(g_{i j}(T)\right) \operatorname{Diag}(T, \ldots, T) .
$$

Observe next that the matrix $\left(f_{i j}(0) 1_{\operatorname{ker} T}\right)$ has the same norm as the matrix $M=\left(f_{i j}(0)\right)$ acting on $\mathbb{C}^{n}$. Moreover, there exists a unit vector $w=\left(\xi_{1}, \ldots, \xi_{n}\right)^{t}$ in $\mathbb{C}^{n}$ such that $\|M w\|=\|M\|$. Now let $x$ be a unit vector in $\operatorname{ker}(T)$ and note that if $s$ is the unit vector

$$
s=\left(\xi_{1} x, \ldots, \xi_{n} x\right)^{t} \in \mathcal{H}^{(n)},
$$

then, from (12), we have $\left(f_{i j}(T)\right) s=\left(f_{i j}(0) 1_{\mathcal{H}}\right) s$. Write $M w=\left(\gamma_{1}, \ldots, \gamma_{n}\right)^{t}$, and observe that

$$
\begin{aligned}
\|M\| & =\|M w\|=\left\|\left(\gamma_{1}, \ldots, \gamma_{n}\right)^{t}\right\|=\left\|\left(\gamma_{1} x, \ldots, \gamma_{n} x\right)^{t}\right\| \\
& =\left\|\left(f_{i j}(0) 1_{\mathcal{H}}\right) s\right\|=\left\|\left(f_{i j}(T)\right) s\right\| \leq\left\|\left(f_{i j}(T)\right)\right\|,
\end{aligned}
$$


which is the desired inequality.

\section{Complete contractivity of $\widetilde{\boldsymbol{\Phi}}$.}

In this section we prove the following analog of Theorem 4.1 for the mapping $\widetilde{\Phi}$, and thus complete the proof of Theorem $1.1 \mathrm{~b}$ ).

Theorem 5.1. For every $T$ in $\mathcal{L}(\mathcal{H})$, the map $\widetilde{\Phi}: \mathcal{A}_{T} \rightarrow \mathcal{A}_{\widetilde{T}}$ defined in Section 1 is completely contractive.

Let $n \in \mathbb{N}$ and let $\left(f_{i j}\right)$ be an arbitrary $n \times n$ matrix with entries from $\operatorname{Hol}(\sigma(T))$. As noted above, we must show that

$$
\left\|\left(f_{i j}(\widetilde{T})\right)\right\| \leq\left\|\left(f_{i j}(T)\right)\right\| .
$$

To establish (13) we need some lemmas. The following lemma will simplify greatly the remainder of the argument:

Lemma 5.2. Suppose $n \in \mathbb{N}$ and $\left(f_{i j}\right)$ is an $n \times n$ matrix with entries from $\operatorname{Hol}(\sigma(T))$. Let $T \in \mathcal{L}(\mathcal{H})$ and suppose that there exists a sequence $\left\{A_{n}\right\}$ in $\mathcal{L}(\mathcal{H})$ such that:

a) $\left\|A_{n}-T\right\| \rightarrow 0$,

b) $\left\|\widetilde{A}_{n}-\widetilde{T}\right\| \rightarrow 0$, and

c) $\left\|\left(f_{i j}\left(\widetilde{A}_{n}\right)\right)\right\| \leq\left\|\left(f_{i j}\left(A_{n}\right)\right)\right\|$ for all $n$ sufficiently large.

Then (13) is satisfied.

Proof. By the upper semicontinuity of the spectrum,

$$
\sigma\left(A_{n}\right) \subset \cap_{i, j=1}^{n}\left(\text { domain } f_{i j}\right)
$$

for $n$ sufficiently large, so $f_{i j}\left(A_{n}\right)$ and $f_{i j}\left(\widetilde{A}_{n}\right)$ are defined for such $n$. Moreover, as noted several times above,

$$
\left\|f_{i j}\left(A_{n}\right)-f_{i j}(T)\right\| \rightarrow 0, \quad\left\|f_{i j}\left(\widetilde{A}_{n}\right)-f_{i j}(\widetilde{T})\right\| \rightarrow 0, \quad i, j=1, \ldots, n .
$$

Since there are only a finite number of functions $f_{i j}$, it follows easily that

$$
\left\|\left(f_{i j}\left(A_{n}\right)\right)-\left(f_{i j}(T)\right)\right\| \rightarrow 0, \quad\left\|\left(f_{i j}\left(\widetilde{A}_{n}\right)\right)-\left(f_{i j}(\widetilde{T})\right)\right\| \rightarrow 0,
$$

and these facts, together with c) above, yield the result.

Lemma 5.3. With the notation as above, if $T=U P$ and $P$ has trivial kernel, then (13) holds.

Proof. Suppose first that $P$ is invertible. By c) of Lemma 2.2,

$$
\begin{aligned}
\left(f_{i j}(\widetilde{T})\right) & =\left(P^{\frac{1}{2}} f_{i j}(T) P^{-\frac{1}{2}}\right) \\
& =\operatorname{Diag}\left(P^{\frac{1}{2}}, \ldots, P^{\frac{1}{2}}\right)\left(f_{i j}(T)\right) \operatorname{Diag}\left(P^{-\frac{1}{2}}, \ldots, P^{-\frac{1}{2}}\right) .
\end{aligned}
$$


Thus, utilizing (4), Lemma 2.2 a), and Theorem 4.1, we obtain

$$
\begin{aligned}
\left\|\left(f_{i j}(\widetilde{T})\right)\right\| & \leq\left\|\left(P f_{i j}(T) P^{-1}\right)\right\|^{\frac{1}{2}}\left\|\left(f_{i j}(T)\right)\right\|^{\frac{1}{2}} \\
& =\left\|\left(f_{i j}(\widehat{T})\right)\right\|^{\frac{1}{2}}\left\|\left(f_{i j}(T)\right)\right\|^{\frac{1}{2}} \\
& \leq\left\|\left(f_{i j}(T)\right)\right\|,
\end{aligned}
$$

as desired. Now let $P$ be an arbitrary quasiaffinity, and let the sequences $\left\{Q_{n}\right\}$ and $\left\{A_{n}\right\}$ be as defined in the proof of Lemma 2.4, so we have a) and b) of Lemma 5.2 satisfied. Since each $\left|A_{n}\right|$ is invertible by construction, by what was just shown,

$$
\left\|\left(f_{i j}\left(\widetilde{A}_{n}\right)\right)\right\| \leq\left\|\left(f_{i j}\left(A_{n}\right)\right)\right\|,
$$

so c) of Lemma 5.2 is satisfied and the result follows from that lemma.

Lemma 5.4. Let $n \in \mathbb{N}$, let $\left(f_{i j}\right)$ be any $n \times n$ matrix with entries from $\operatorname{Hol}(\sigma(T))$ and suppose $T=U P$ is any operator in $\mathcal{L}(\mathcal{H})$ such that

$$
\operatorname{dim}\left(\operatorname{ker} U^{*}\right) \geq \operatorname{dim}(\operatorname{ker} U)>0 .
$$

Then (13) is satisfied.

Proof. Let the sequence $\left\{A_{n}\right\}_{n=1}^{\infty}$ be as defined in Lemma 2.5, and observe that from the proof of that lemma, we know that a) and b) of Lemma 5.2 are satisfied. Moreover, since each $\left|A_{n}\right|$ is a quasiaffinity, Lemma 5.3 yields that c) of Lemma 5.2 is satisfied, and the result follows from Lemma 5.2.

In view of the discussion preceding Lemma 2.6, the proof of Theorem 5.1 (and thus the proof of Theorem $1.1 \mathrm{~b}$ ) is completed by the following:

Lemma 5.5. For every $T=U P$ in $\mathcal{L}(\mathcal{H})$ such that $U$ is a nonunitary coisometry, for every $n \in \mathbb{N}$, and for every $n \times n$ matrix $\left(f_{i j}\right)$ with entries from $\operatorname{Hol}(\sigma(T))$, (13) is satisfied.

Proof. Let the sequence $\left\{S_{n}\right\}_{n=1}^{\infty}$ be as defined in the proof of Lemma 2.6, and observe from that proof that $\left\|S_{n}-(\widehat{T})^{*}\right\| \rightarrow 0$ and $\left\|\widetilde{S}_{n}-(\widetilde{T})^{*}\right\| \rightarrow 0$. Moreover, since $\left|S_{n}\right|$ is an isometry for each $n$, Lemma 5.4 applies to give that

$$
\left\|\left(\widetilde{f}_{i j}\left(\widetilde{S}_{n}\right)\right)\right\| \leq\left\|\left(\widetilde{f}_{i j}\left(S_{n}\right)\right)\right\|,
$$

so that a), b) and c) of Lemma 5.2 are satisfied (with $S_{n} \rightarrow A_{n}$ (i.e., $S_{n}$ replaces $\left.A_{n}\right), \widehat{T}^{*} \rightarrow T, \widetilde{T}^{*} \rightarrow \widetilde{T}$, and $\widetilde{f}_{i j} \rightarrow f_{i j}$ ), so

$$
\left\|\left(\widetilde{f}_{i j}\left(\widetilde{T}^{*}\right)\right)\right\| \leq\left\|\left(\widetilde{f}_{i j}\left(\widehat{T}^{*}\right)\right)\right\| .
$$

Upon taking adjoints in (14) and using Theorem 4.1, the result follows. 
Of course, one reason for establishing that the maps $\widetilde{\Phi}$ and $\widehat{\Phi}$ are completely contractive is that the extension theorems of Arveson and Stinespring can be applied to obtain the structure of such maps (cf., e.g., [8]), and thus we get the following:

Theorem 5.6. Let $T$ be an arbitrary operator in $\mathcal{L}(\mathcal{H})$, and let $\widetilde{\Phi}$ and $\widehat{\Phi}$ be the maps defined in Theorem 1.1. Then there exist Hilbert spaces $\widetilde{\mathcal{K}}=\widetilde{\mathcal{K}}_{T}$ and $\widehat{\mathcal{K}}=\widehat{\mathcal{K}}_{T}$ containing $\mathcal{H}$, and $C^{*}$-homomorphisms $\widetilde{\Psi}: C^{*}(T) \rightarrow \mathcal{L}(\widetilde{\mathcal{K}})$ and $\widehat{\Psi}: C^{*}(T) \rightarrow \mathcal{L}(\widehat{\mathcal{K}})$ (where $C^{*}(T)$ is the smallest unital $C^{*}$-algebra containing $\left.\mathcal{A}_{T}\right)$ such that for every $f$ in $\operatorname{Hol}(\sigma(T))$,

$$
\widetilde{\Phi}(f(T))=\left.P_{\mathcal{H}} \widetilde{\Psi}(f(T))\right|_{\mathcal{H}}
$$

and

$$
\widehat{\Phi}(f(T))=\left.P_{\mathcal{H}}^{(2)} \widehat{\Psi}(f(T))\right|_{\mathcal{H}},
$$

where $P_{\mathcal{H}}^{(1)}$ and $P_{\mathcal{H}}^{(2)}$ are the orthogonal projections of $\widetilde{\mathcal{K}}$ and $\widehat{\mathcal{K}}$, respectively, onto $\mathcal{H}$.

The implications of Theorem 5.6 for the Aluthge and Duggal transforms will be the subject of a forthcoming paper by the authors.

Acknowledgement. The second and third authors were supported by KOSEF Research Project No. R01-2000-00003. The fourth author acknowledges the support of the National Science Foundation.

\section{References}

[1] A. Aluthge, On p-hyponormal operators for $0<p<1$, Integral Equations Operator Theory, 13 (1990), 307-315, MR 91a:47025, Zbl 0718.47015.

[2] A. Brown and C. Pearcy, Introduction to Operator Theory I, Springer Verlag, New York, 1977, MR 58 \#23463, Zbl 0371.47001.

[3] E. Heinz, Beiträe zur Störungstheorie der Spektralzerlegung, Math. Ann., 123 (1951), 415-438, MR 13,471f, Zbl 0043.32603.

[4] I. Jung, E. Ko and C. Pearcy, Aluthge transforms of operators, Integral Equations Operator Theory, 37 (2000), 437-448, MR 2001i:47035.

[5] - Spectral pictures of Aluthge transforms of operators, Integral Equations Operator Theory, 40 (2001), 52-60, MR 2002b:47007.

[6] The iterated Aluthge transform of an operator, Integral Equations Operator Theory, to appear.

[7] B. Sz.-Nagy and C. Foiaş, Harmonic Analysis of Operators on Hilbert Space, NorthHolland, Amsterdam, 1970, MR 43 \#947, Zbl 0201.45003.

[8] V. Paulsen, Completely Bounded Maps and Dilations, Longman Sci. \& Tech. Pittman Research Note 146, 1986, MR 88h:46111, Zbl 0614.47006. 
[9] C. Pearcy, Some Recent Developments in Operator Theory, C.B.M.S. Regional Conference Series in Mathematics, 36, Amer. Math. Soc., Providence, 1978, MR 58 \#7120, Zbl 0444.47001.

[10] T. Yamazaki, On numerical range of the Aluthge transformation, Linear Algebra and Applications, 341 (2002), 111-117, MR 2003a:47012.

Received April 4, 2002.

Department of Mathematics

TeXas A\&M University

College Station, TX 77843

E-mail address: foias@math.tamu.edu

Department of Mathematics

KYUNGPOOK NATIONAL UNIVERSITY

TAEgu 702-701

KOREA

E-mail address: ibjung@kyungpook.ac.kr

Department of Mathematics

EWha Women's University

SEOUL 120-750

KoreA

E-mail address: eiko@mm.ewha.ac.kr

Department of Mathematics

TEXAS A\&M UNIVERSITY

College Station, TX 77843

E-mail address: pearcy@math.tamu.edu 